Publisher's note: Springer Nature remains neutral with regard to jurisdictional claims in published maps and institutional affiliations.

\section{REFERENCES}

1. Christopoulos A, Changeux JP, Catterall WA, Fabbro D, Burris TP, Cidlowski JA, et al. International Union of Basic and Clinical Pharmacology. XC. Multisite pharmacology: recommendations for the nomenclature of receptor allosterism and allosteric ligands. Pharmacol Rev. 2014;66:918-47.

2. Huidobro-Toro JP, Valenzuela $C F$, Harris RA. Modulation of $G_{A B A}$ receptor function by $\mathrm{G}$ protein-coupled $5-\mathrm{HT}_{2 \mathrm{C}}$ receptors. Neuropharmacology. 1996:35:1355-63.
3. Im WB, Chio CL, Alberts GL, Dinh DM. Positive-allosteric modulator of the human 5$\mathrm{HT}_{2 \mathrm{C}}$ receptor. Mol Pharmacol. 2003;64:78-84.

4. Ding C, Bremer NM, Smith TD, Seitz PK, Anastasio NC, Cunningham KA, et al. Exploration of synthetic approaches and pharmacological evaluation of PNU$69176 \mathrm{E}$ and its stereoisomer as $5-\mathrm{HT}_{2 \mathrm{C}}$ receptor allosteric modulators. ACS Chem Neurosci. 2012;3:538-45.

5. Wild CT, Miszkiel JM, Wold EA, Soto CA, Ding C, Hartley RM, et al. Design, synthesis, and characterization of 4-undecylpiperidine-2-carboxamides as positive-allosteric modulators of the serotonin (5-HT) 5- $\mathrm{HT}_{2 \mathrm{C}}$ receptor. J Med Chem. $2018 \mathrm{https}: / /$ doi. org/10.1021/acs.jmedchem.8b00401

6. Garcia-Carceles J, Decara JM, Vazquez-Villa H, Rodriguez R, Codesido E, Cruces J, et al. A positive-allosteric modulator of the serotonin $5-\mathrm{HT}_{2 \mathrm{C}}$ receptor for obesity. $\mathrm{J}$ Med Chem. 2017;60:9575-84.

\title{
Motion mapping in humans as a biomarker for psychiatric disorders
}

\author{
Ipsit V. Vahia ${ }^{1,2}$ and Brent P. Forester ${ }^{1,2}$ \\ Neuropsychopharmacology (2019) 44:231-232; https://doi.org/10.1038/s41386-018-0205-7
}

Measuring movement has been a cornerstone of studying behavior in animals in controlled studies.

Movement data have served as a primary window into behavior, and by proxy, social function, mood, and cognition [1]. In humans, variations in locomotor activity have served as markers of a range of psychiatric syndromes including major depression, bipolar disorder, anxiety, catatonia, and substance use disorders [2]. Reports of sensor-based motion measurement in humans date back to the 1950s [2], and motion-based models of human psychopathology have been key to developing animal models of psychopathology [1]. A vast literature has documented how these animal models have facilitated development of motion-based signatures of antidepressant, anxiolytic, and other drug effects and guided drug development [3]. However, the limitations of technology thus far have meant that motion-mapping in humans has remained largely restricted to experimental settings.

Phenotyping of naturalistic human behavior continues to depend on self-report or observer-report measures, with sampling often at intervals of hours to days. Newer technologies, supported by advances in wireless connectivity, more compact and reliable sensors and devices, and higher computing power can now sample movement at intervals of seconds, and can facilitate measurement of human motion in the natural living environment in ways previously not possible [4]. Such technologies are setting the stage for movement to become a major new phenotypic biomarker in neuropsychiatry.

A wide array of validated technologies can quantify motion. These range from active sensors that move along with the body (e.g., accelerometers, gyroscopes), passive "line of sight" technologies (e.g., cameras, thermal sensors, and lasers), passive technologies that can detect motion through walls (e.g., radio wave sensors) and positioning technologies (e.g., GPS, satellite imaging) [5]. It is now possible to track individual contractions of facial muscles (used in computer vision and analysis of micro emotions), complex motions such as smoking or drinking coffee
[6] or even the movement of an individual across a global geographic field. Work in this new emerging field necessitates collaborative teams with a range of expertise that includes hardware development, signal processing, big data processing and analytics, machine learning, data visualization, and clinical implementation.

Early work in this field has demonstrated feasibility of translating this intensive sensor-based approach to clinical research. As an example, our group has demonstrated that by measuring how low frequency radio signals bounce off the human body and objects in a defined space (up to 800 sq.ft.), it is possible to elicit gait speed, gait patterns and spatial location. Using clinical correlation, we have established that these data can serve as markers of apathy, pacing, and disrupted circadian rhythms in patients with dementia [5].

A marker of the maturing of this field is recent $\mathrm{NIH}$ investment into major collaborative initiatives such as the MD2K initiative [7], which supports the acceleration of translating motion sensor data into validated biomarkers of behavior. While the need for privacy and security around sensor data is well recognized, innovative solutions around secure data storage and transfer will be crucial to this approach gaining widespread acceptance and use [4].

\section{ACKNOWLEDGEMENTS}

The authors acknowledge the contributions of Patrick Monette to the preparation of this manuscript.Competing interests: Dr. Forester has received Research Grant Support from Eli Lilly, Biogen, Rogers Family Foundation and the National Institutes of Health; Dr. Vahia has received Research Grant Support from the Once Upon a Time Foundation, the Massachusetts Institute of Technology and the National Institutes of Health

Publisher's note: Springer Nature remains neutral with regard to jurisdictional claims in published maps and institutional affiliations. 


\section{REFERENCES}

1. Henry BL, Minassian A, Young JW, Paulus MP, Geyer MA, Perry W. Cross-species assessments of motor and exploratory behavior related to bipolar disorder. Neurosci Biobehav Rev. 2010;34:1296-306. https://doi.org/10.1016/j.neubiorev.2010.04.002

2. Teicher MH. Actigraphy and motion analysis: new tools for psychiatry. Harv Rev Psychiatry. 1995;3:18-35.

3. Tecott LH, Nestler EJ. Neurobehavioral assessment in the information age. Nat Neurosci. 2004;7:462-6. https://doi.org/10.1038/nn1225

4. Andreu-Perez J, Poon CC, Merrifield RD, Wong ST, Yang GZ. Big data for health. IEEE J Biomed Health Inform. 2015;19:1193-208. https://doi.org/10.1109/ JBHI.2015.2450362
5. Collier S, Monette P, Hobbs K, Tabasky E, Forester BP, Vahia IV. Mapping movement: applying motion measurement technologies to the psychiatric care of older adults. Curr Psychiatry Rep. 2018;20:64 https://doi.org/10.1007/s11920-0180921-z

6. Shoaib M, Bosch S, Incel OD, Scholten H, Havinga PJ. Complex human activity recognition using smartphone and wrist-worn motion sensors. Sensors. 2016;16:426 https://doi.org/10.3390/s16040426

7. Kumar S, Abowd G, Abraham WT, al'Absi M, Chau DHP, Ertin E, et al. Center of excellence for mobile sensor data-to-knowledge (MD2K). IEEE Pervasive Comput. 2017;16:18-22. https://doi.org/10.1109/MPRV.2017.29

\title{
Treating cue-reactivity with brain stimulation: a new (transdiagnostic) approach
}

\author{
Colleen A. Hanlon ${ }^{1,2,3}$ \\ Neuropsychopharmacology (2019) 44:232-233; https://doi.org/10.1038/s41386-018-0215-5
}

The struggle between internal self-control and external temptation from environmental cues is a tale as old as written history, yet as relevant today as any time in the past. Just as Homer wrote about Odysseus and the seductive Siren Songs (800 B.C.), or Tintorreto painted Adam's temptation in the Garden of Eden (1551 A.D.), twenty-first century depictions of life frequently highlight the struggle to maintain focus despite proverbial "apples" that interrupt our journey. For most individuals, the occasional surrender to a tempting cue will not impair their ability to fulfill daily and longer term responsibilities. For other individuals, however, elevated reactivity to positive or negative cues causes a disabling cascade of events ultimately impeding long-term goals. Elevated cue-reactivity is also a prominent feature of alcohol and substance-use disorder, posttraumatic stress disorder (PTSD), and obsessive behavior disorders, such as eating and gambling.

In these populations, salient cues evoke elevated activity in a consistent network of neural regions: the ventral medial prefrontal cortex (MPFC), anterior cingulate cortex (ACC), and insula. This network may be thought of as a "transdiagnostic neural biomarker" for cue-reactivity. In substance-abuse literature, meta-analyses have demonstrated that these regions are reliably activated by drug cues and may predict relapse [1, 2]. In a recent study by our group, 156 substance dependent individuals performed a drug cue-exposure task tailored to their drug of choice (55 cocaine, 53 alcohol, 48 nicotine) [3]. Multivariate kmeans clustering revealed three distinct clusters of elevated activity when the participants were viewing the drug cues vs. neutral non-drug cues: the MPFC/ACC, the left inferior frontal gyrus/insula, and the right premotor cortex.

From a therapeutic perspective, novel non-invasive brain stimulation treatment protocols are being designed to target the MPFC-ACC-Insula circuit directly [4]. In the cue-reactivity study described above, cortical projection analysis revealed that the frontal pole (FP) was the cortical location closest to the maximal number of significant cue-reactivity clusters. A recent shamcontrolled study in 49 individuals demonstrated that continuous theta burst stimulation (TBS) - a particularly potent and efficient form of transcranial magnetic stimulation (TMS) - directed to the left FP decreases drug cue-reactivity among heavy alcohol users and cocaine users [5]. This protocol also decreases functional connectivity in this MPFC/ACC/Insula network [6].

FP TMS is also being used to improve cue-reactivity in PTSD and obsessive behavioral disorders. Dr Rebecca Price and colleagues at the University of Pittsburg, e.g., are currently evaluating FP TBS, as a tool to decrease compulsive behaviors in obsessive compulsive disorder, many of which are cue-evoked (NCT \#03265015). The use of this MPFC-ACC-Insula network as a framework for modulating cue-reactivity is just beginning. Although there will be several challenges associated with developing TMS strategies to modulate this network (e.g., reaching these deep targets, disease-tailored protocols), the MPFC-ACC-Insula network appears to be a fruitful and transdiagnostic neural biomarker to explore for next generation brain stimulation protocols.

\section{FUNDING}

Dr. Hanlon's research has been funded by the National Institute of Health. Dr. Hanlon receives compensation as a consultant for Brain Research \& Development Services.

\section{ADDITIONAL INFORMATION}

\section{REFERENCES}

1. Janes AC, Pizzagalli DA, Richardt $S$, de BFB, Chuzi S, Pachas $G$, et al. Brain reactivity to smoking cues prior to smoking cessation predicts ability to maintain tobacco abstinence. Biol Psychiatry. 2010;67:722-9.

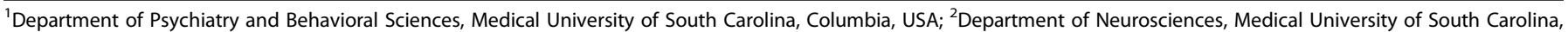
Columbia, USA and ${ }^{3}$ Center for Biomedical Imaging, Medical University of South Carolina, Columbia, USA

Correspondence: Colleen A. Hanlon (hanlon@musc.edu)
}

Published online: 7 October 2018 